\title{
HACIA UNA EPISTEMOLOGÍA DE LA COMUNICACIÓN EN LA POSTMODERNIDAD
}

\section{TOWARDS A COMMUNICATION EPISTEMOLOGY IN POSTMODERNITY}

\author{
Amaro La Rosa*
}

\begin{abstract}
RESUMEN
El artículo formula algunas propuestas epistemológicas sobre el estatus científico de la comunicación en la posmodernidad, incidiendo especialmente en la comunicación masiva y en su vinculación con el enfoque transdisciplinario.
\end{abstract}

\section{PALABRAS CLAVE:}

Postmodernidad, comunicación, epistemología, transdisciplinareidad

\begin{abstract}
The article makes some epistemological proposals about scientific status of communication in postmodernity, focusing in mass communication and their relationship with the transdisciplinary approach.
\end{abstract}

\section{KEY WORDS:}

Postmodernity, communication, epistemology, transdisciplinarity 


\section{Postmodernidad}

Como punto de partida para este análisis, luego de la revisión de los criterios de diversos autores comprendemos que la postmodernidad es un concepto que tiene que ver con la sociedad mientras que el postmodernismo está ligado más que nada con la cultura en sus diversas manifestaciones.

Para Lyotard (1984) en la posmodernidad lo único cierto es el cambio constante, rasgo que podemos observar en las últimas décadas a nivel mundial. Han caducado los viejos criterios de legitimación en medida tal que se pasa de la noción valorativa de lo justo o lo verdadero a formularse la pregunta pragmática ¿para qué sirve? Igualmente se admiten como plenamente valederos criterios antes contrapuestos, tal como sucede con el constructivismo que une tanto en la teoría como en la práctica dos puntos de vista que antes se consideraban Irreconciliables: Piaget y Vigotsky. Estima Lyotard que en la posmodernidad de manera creciente las experiencias están mediadas por la tecnología, tal como podemos comprobarlo si observamos los gadgets y artefactos que cotidianamente empleamos.

Lyotard entiende asimismo que en la postmodernidad se pone en evidencia una concepción distinta de la relación entre el ser humano y la realidad, la cual concibe que ésta no es algo dado per se sino que se construye. De modo similar Kenneth Gergen (2011) estima que los fenómenos sociales no existen realmente como hechos de la realidad pues son construidos por la actividad social humana. Asimismo plantea que de los procesos sociales surgen los significados que se van construyendo durante la interacción.
Con respecto al paradigma posmoderno Coca y Valero (2010) afirman que en la posmodernidad la racionalidad de la ciencia defiende la incertidumbre permanente y es por ello que se requiere una búsqueda de la verdad que permita controlar la incertidumbre.

\section{Epistemología de la comunicación y postmodernidad}

La epistemología busca respuestas a la multiplicidad de interrogantes que se formulan con respecto al conocimiento y en lo que nos compete en relación con el estatus científico de la Comunicación. La comunicación es una actividad humana que ha evolucionado a través de los tiempos desde las formas más primitivas de intercambio, donde probablemente existían muchas similitudes con la comunicación animal, hasta las modalidades más avanzadas de representación simbólica de la realidad. Por sí misma la comunicación, como cualquier otro fenómeno de la realidad, no involucra un conocimiento científico, pero como fruto de la reflexión, y de la importancia que adquieren, en especial las diversas formas de comunicación masiva, se han ido acumulando en las últimas décadas conocimientos que paso a paso han permitido que se conforme una disciplina cuyo status de ciencia aún se discute. A pesar de ser relativamente joven, la Comunicación ha experimentado un acelerado desarrollo, resultado de la incesante investigación y de la construcción teórica.

En un trabajo previo (La Rosa, 2007) afirmábamos que la diversidad de puntos de vista sobre la naturaleza de la Comunicación como disciplina, las podemos ubicar en una escala en la cual incluiremos groso modo a: 
1. Los comunicólogos: Quienes están convencidos que la comunicación responde a los estándares del conocimiento científico, sosteniendo en consecuencia que maneja criterios conceptuales propios los cuales son fruto de la investigación y que por tanto posee la categoría de ciencia.

2. Los integracionistas: Quienes piensan que la Comunicación es un cuerpo de conocimientos que no tiene identidad como ciencia. Ello porque integra elementos conceptuales de diversas disciplinas sociales e igualmente porque solamente aplica -sin mayor aporte propio-, los criterios metodológicos desarrollados por las Ciencias Sociales, más legitimadas en el campo de la investigación.

3. Los negativistas: Quienes estiman que los estudios comunicacionales no reúnen las condiciones suficientes para poderlos denominar científicos, pues constan solamente de temáticas dispersas analizadas desde paradigmas diversos.

Sin desarrollar el criterio por el momento podemos afirmar categóricamente que existe un cuerpo de conocimientos bastante amplio sobre la comunicación. Sin embargo, aquí existe un escollo que es necesario advertir. Están por un lado los conocimientos derivados de la investigación pura y aplicada de especialistas cuya obra se difunde abiertamente en la comunidad científica. Pero por otro hay un amplio número de estudios que solamente se registran en informes técnicos, cuyos resultados y aplicabilidad están regulados por criterios de confidencialidad determinados por los organismos privados e instituciones internacionales que los financian.
Tal como lo afirmáramos de modo reiterado en algunos trabajos nuestros (La Rosa, 1999, 2001, 2003) estamos convencidos que un problema epistemológico de partida para el análisis de nuestro objeto de estudio radica en los términos que usamos al intentar responder a la pregunta ¿cuál es el objeto de estudio de la Comunicación?

Es necesario anotar que bajo la aparentemente simple palabra comunicación involucramos fenómenos de diverso orden a los cuales deberíamos tratar de acuerdo a sus características específicas. No existe por consiguiente un modelo universal, que pudiera ser utilizado para las circunstancias de comunicación más diversas. Nos encontramos con paradigmas que utilizan connotaciones propias, las cuales es menester manejar adecuadamente. En el caso de la comunicación masiva teorizar resulta una tarea marcadamente compleja por las siguientes razones:

- Los profundos cambios producidos en la sociedad que se reflejan a su vez en modificaciones de mayor o menor magnitud en las modalidades de comunicación. En este sentido cabría revisar, los criterios que de una u otra manera relacionan la historia de la humanidad con la comunicación (v.g. Mc Luhan, Toffler, Vattimo, Burcet).

- La digitalización de las comunicaciones, que hace posible utilizar diversos soportes para la trasmisión de mensajes.

- El acelerado desarrollo de la tecnología y en especial de la informática que ha propiciado la convergencia de las telecomunicaciones.

- El desarrollo incesante de los medios de comunicación que producen fenómenos de creciente complejidad. 
- La fugacidad de sus manifestaciones que convierte en problemas de estudio lo ayer inexistente, o hace perder rápidamente la vigencia de las temáticas. Se ha dicho insistentemente que los fenómenos de la comunicación cambian de manera tan acelerada que la investigación no puede recoger todo lo que ocurre, por cuanto apenas le está tomando atención, ya se modificaron profundamente las cosas. Pensamos sin embargo sobre el particular que muchas veces los hechos sociales que son objeto de la investigación de la comunicación condicionan estudios ex post facto, y que esta relativa permanencia de la temática no descalifica su análisis.

- Las modificaciones que se han producido en las modalidades de producción de los medios masivos así como el surgimiento de medios, que involucran nuevas formas de comunicación y de audiencia. Usando palabras de Cadenas (2012) en la actualidad la audiencia desempeña simultáneamente el rol de receptores audiencias y productores emisores.

- Las variaciones constantes que se producen en las necesidades y percepciones de la audiencia.

- Las nuevas modalidades de consumo de los medios, en especial de los interactivos que ahora otorgan mayor poder de decisión al perceptor.

- Los logros de la investigación aplicada, que a pesar de demostrar su utilidad merecen eventualmente ciertas críticas en círculos académicos por su falta de rigor. Al respecto afirma Sanz (1987) que es imposible pedir verdades a prueba de errores, pero lo cierto es que éstos tienen la posibilidad de corregirse y que las verdades de hoy pueden mañana ser modificadas.
En la ciencia, los paradigmas orientan el quehacer investigativo, desde la observación hasta el análisis y la interpretación de los resultados. De acuerdo al paradigma orientador de su percepción del mundo, el especialista "verá" la realidad desde "su propia mirada" científica, que ha desarrollado como consecuencia de la praxis de su especialización. Para decirlo en otros términos, el científico observará los fenómenos, los cuales posteriormente describirá y explicará, en consonancia con su criterio de lo que significa la realidad.

En relación con varios de los aspectos previamente mencionados, percibimos una crisis de los paradigmas de la comunicación que ha removido los cimientos de algunos aparentemente inamovibles enfoques teóricos, obligando a reformulaciones, renovación de criterios y la urgente demanda de nuevos modelos.

Desde una perspectiva que consideramos extrema Drugus( 2010) entiende que en la posmodernidad ya resulta obsoleto el propio concepto de ciencia que estima como uno de los mitos de la modernidad. Sugiere que usar este concepto en la posmodernidad sería algo similar a "estudiar el sistema planetario a partir de la teoría geocéntrica" (íb, p.56). Plantea como más apropiados los términos investigación o enfoque.

Vattimo (2003) plantea algunas apreciaciones en torno a la sociedad posmoderna que cabe analizar por sus implicancias para el tema de este acápite. Se trata de la sociedad de la comunicación generalizada donde existen un sinnúmero de recursos de comunicación entre los seres humanos. La ampliación de las posibilidades de información promueve su ampliación constante en ligazón estrecha con las 
leyes del mercado, lo que se expresa por ejemplo en los grandes conglomerados que unen medios en diversos soportes: radio, televisión, prensa, medios online. Plantea igualmente un rasgo que ahora estimamos se hace realidad de manera alarmante: La sociedad ha olvidado lo que significa la transparencia y la intimidad donde la vida privada es vida de todos. Talk shows de diversa naturaleza hurgan si escrúpulo alguno en aspectos íntimos de la vida personal, desde luego con la anuencia de sus protagonistas.

Los medios no pueden apartarse de la realidad de la sociedad postmoderna en la cual funcionan. Para Fernández y Franco (2010, p. 124) sostienen que las prácticas comunicativas mediáticas reproducen un modelo de producción y comprensión, de construcción y deconstrucción racional del conocimiento de la realidad Según Aguado (2009) las operaciones del sistema de medios de comunicación, que se vincula con otros sistemas sociales, se orientan por dos clases de códigos (interés/no interés y relevancia/opacidad). Como resultante de las selecciones que determinan se configuran los temas que el medio trata a los cuales el autor denomina estructuras situadas de significado. Esto nos explica el porqué en la actualidad se dan similitudes en el tratamiento de la realidad por parte de los medios.

\section{Comunicación y Transdisciplinariedad}

Para Wolfeneden (2009) la transdisciplinareidad supone una visión integrada de la realidad en la cual confluyen los criterios de disciplinas diversas para la apreciación de la realidad de manera holística.

En el gráfico 1. Intentamos una representación de los tres pilares epistemológicos de la transdisciplinareidad (Max-Neef, 2005).

\section{Gráfico 1}

\section{TRES PILARES DE LA TRANSDISCIPLINAREIDAD}

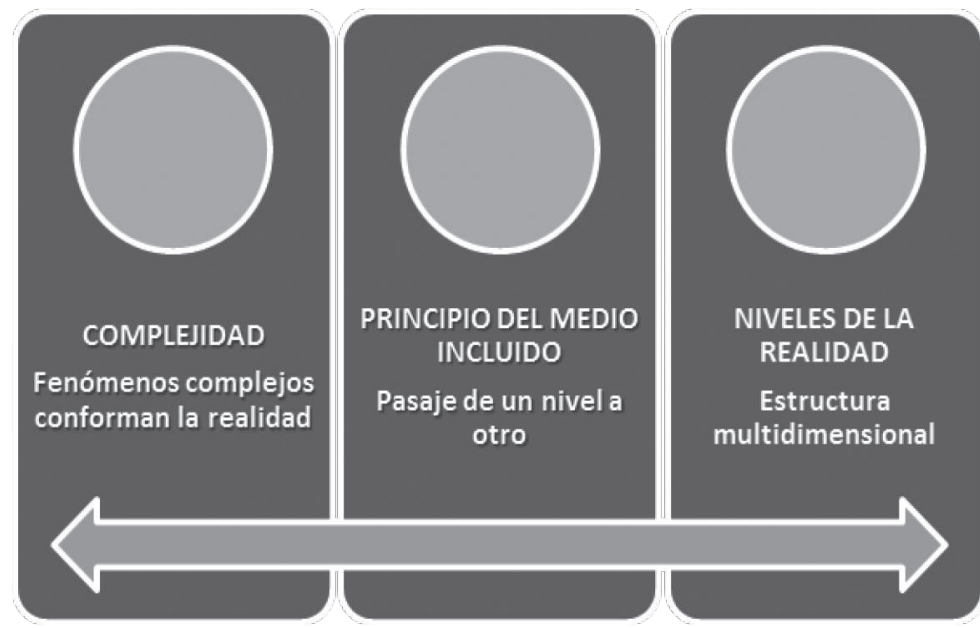

Basado en Max- Neef (2005). Elaboración: ALR 
Para Drugus (2010) el mundo posmoderno exige el conocimiento transdisciplinario en la medida en que éste reconoce que vivimos en un mundo complejo, incierto y pluralista. Desde la perspectiva de García (2006) el enfoque transdisciplinario intenta articular ciencias y conocimientos. A base de la confrontación de las disciplinas obtiene nuevos datos que articula entre sí de manera holística.

La naturaleza de la comunicación masiva en la sociedad postmoderna demanda para nosotros la necesidad de analizarla desde la perspectiva transdisciplinaria. Ahora bien, a decir de Berlanga (2012) las transferencias de significado que se producen en la posmodernidad, afectan las prácticas comunicativas. En este sentido afirma: "La filosofía de la Web 2.0, donde priman la participación y la colaboración, la obra colectiva, el carácter público y abierto con todos los entresijos teóricos y prácticos que comportan estas manifestaciones, es el fruto genuino de este contexto de posmodernidad"(íbid, p. 122). Si quisiéramos aplicar este criterio a la realidad, es evidente lo que sucede en el terreno de los teléfonos móviles que han transformado las modalidades de comunicación y cuya penetración ha llegado a tal nivel que en muchas ciudades del mundo, tal como ocurre en Lima, han superado largamente el número de habitantes.

En una entrevista realizada por Charlie Rose al creador de Mosaic, Netscape y Ning Mark Andreessen, éste afirmó que en la posmodernidad la obsolescencia tecnológica es muy rápida, de tal manera que a veces comentamos lo sucedido hace menos de una década y nos parece que habláramos de un pasado lejano. Y esto es muy cierto, pues si nos referimos a los recursos tecnológicos encontramos que permanentemente están produciéndose nuevas versiones, en medida tal que debemos descartar algo a lo que aún no hemos terminado de sacarle partido. Este es el caso del hardware: Parece lejano en el tiempo el Windows 2000 y al redactar estas líneas ya se ponía en el mercado el Windows 8 que dejaría de lado al Windows 7 que se lanzó en el "lejano" octubre de 2009. Por cierto que parece ya parte de la prehistoria el primer sistema operativo Windows que Microsoft comenzó a vender en 1985.

\section{REFERENCIAS:}

AGUADO, J. (2009) Self-observation, self-reference and operational coupling in social systems: steps towards a coherent epistemology of mass media Empedocles European Journal for the Philosophy of Communication, Volume 1, Number 1, 59-74

BERLANGA, I. (2012) Las redes sociales online: Nuevas ágoras de la democracia posmoderna. En TELOS, 92, 118-126

CADENAS, M. (2012) Reflexiones epistemológicas en torno a la interactividad en la era digital. Ponencia presentada en el $14^{\circ}$ Encuentro Latinoamericano de Facultades de Comunicación Social, Lima, octubre

DRUGUS, L. (2010) From modern disciplinary "science" to posmodernity transdiciplinary hnowledge Economy transdisciplinary cognition, Vol XIII (2), 53-69. Revisado 2 de Julio de 2012. Disponible http://www.ugb.ro/etc/etc2010no2/s0105\%20\%282\%29.pdf

FERNANDEZ, S. y FRANCO, A. (2010) Fundamentos epistemológicos para un modelo de comunicación en 
situaciones de conflicto. Utopía y praxis latinoamericana, Año $15, \mathrm{~N}^{\circ}$ 51,pp. 113-125.

GARCÍA, M. (2006) Un Nuevo Desafío en la Investigación: Enfoque transdisciplinario en Comunicación y Desarrollo. Razón y Palabra, F ebrero-Marzo. Disponible http://www.razonypalabra.org.mx/ anteriores/n49/mgarcia.html

LA ROSA, A. (1999) Los desafíos epistemológicos de la investigación de la comunicación en la era del ciberespacio Ponencia presentada en el Seminario Internacional "Tendencias y retos de la investigación en comunicación en América Latina" Lima: PUC/FELAFACS

LA ROSA, A. (2001) Investigación de la comunicación y autopistas de la información. Revista COMUNIFE, año 1, No 1
LA ROSA, A. (2003) Teoría de la Comunicación Lima: Universidad Inca Garcilaso de la Vega.

LA ROSA, A. (2007) Claves para una comprensión de la Comunicación. Revista CONSENSUS de la UNIFE, Volumen 12, No 1, 53-64

LYOTARD, J. (1984) The Postmodern Condition: A report on knowledge Manchester: Manchester University Press

MAX-NEEF, M (2005) Foundations of transdisciplinarity Ecological Economics, 53, 5 - 16.Disponible: http://www.max-neef.cl/download/ Max_Neef_Foundations_of_ transdisciplinarity.pdf

SANZ, J. (1987) Introducción a la Ciencia Lima: Ediciones Amaru. 